\title{
New insights in the Fontan circulation: 4-dimensional respiratory- and ECG-triggered phase contrast magnetic resonance imaging
}

\author{
Christopher Hart ${ }^{1 *}$, Dominik Daniel Gabbert ${ }^{1}$, Inga Voges ${ }^{1}$, Michael Jerosch-Herold², Ana Andrade ${ }^{1}$, Minh Pham', \\ Traudel Hansen ${ }^{1}$, Hans-Heiner Kramer $^{1}$, Carsten Rickers ${ }^{1}$
}

From 16th Annual SCMR Scientific Sessions

San Francisco, CA, USA. 31 January - 3 February 2013

\section{Background}

Evaluation of blood flow characteristics in total cavo-pulmonary connection (TCPC) with CMR remains difficult due to its strong modulation by respiration, and is not yet entirely understood. New approaches using 4D phase contrast magnetic resonance imaging (4D PC MRI) are promising and can contribute to the understanding of hemodynamics in the Fontan Circulation. Our objective was to compare flow, velocities, wall shear stress (WSS) and circulation in the TCPC using respiratory- and ECG triggered 4D PC MRI.

\section{Methods}

10 children with hypoplastic left heart syndrome were evaluated after surgical completion of the Fontan circulation (TCPC with lateral intra-atrial tunnel) in a single center. In all patients one respiratory- (80 -100 phases) and one ECG-triggered (30 phases) 4D PC MRI covering the whole thorax, voxel size ranging from isotropic 1.5 to 2.0 $\mathrm{mm}$ were acquired during a single CMR examination with a custom-made electronic respiratory triggering compatible with our scanner. Dedicated commercial and custom software was used for further analysis of flow, velocity, WSS and circulation.

\section{Results}

Respiratory-triggered acquisitions revealed significantly higher maximum and lower minimum flow, maximum and minimum velocity, maximum WSS, and maximum circulation in the inferior vena cava and tunnel, compared to ECG-triggered 4D PC MRI. Flow, velocity, WSS and circulation in the superior vena cava, and also the flow distribution to the left and right pulmonary arteries showed no differences between the two acquisition modes.

\section{Conclusions}

Respiration-triggered 4D PC MRI of the TCPC avoids averaging of flow, velocity, and WSS over the respiratory cycle, resulting in significant differences to solely ECGtriggered acquisitions. This study suggests that hemodynamics in the TCPC are mainly dependant on respiration, while ventricular function causes only minor modulation of flows in the TCPC connection. This may have potential clinical implications for judging the quality of a TCPC, for example in the failing Fontan, by 4D PC MRI. The data from the respiratory triggered sequences can further add to the understanding of hemodynamics and fluid mechanics in the Fontan circulation.

\section{Funding}

None.

Author details

${ }^{1}$ Pediatric Cardiology, Universitaetsklinik Kiel, Kiel, Germany. ${ }^{2}$ Radiology, Brigham and Women's Hospital, Boston, MA, USA.

Published: 30 January 2013

doi:10.1186/1532-429X-15-S1-038

Cite this article as: Hart et al: New insights in the Fontan circulation: 4-dimensional respiratory- and ECG-triggered phase contrast magnetic resonance imaging. Journal of Cardiovascular Magnetic Resonance 201315 (Suppl 1):O38.

${ }^{1}$ Pediatric Cardiology, Universitaetsklinik Kiel, Kiel, Germany

Full list of author information is available at the end of the article

(c) 2013 Hart et al; licensee BioMed Central Ltd. This is an Open Access article distributed under the terms of the Creative Commons Attribution License (http://creativecommons.org/licenses/by/2.0), which permits unrestricted use, distribution, and reproduction in any medium, provided the original work is properly cited. 